\title{
Metaphor: A Creative Thinking Model for Web Designers
}

\author{
Kay Youn \\ Seton Hill University, Greensburg, Pennsylvania, USA
}

\begin{abstract}
Metaphors have been used in web user interface designs from the earliest stages of the web. As technology has evolved into various web fields, metaphors have become even more useful and at the same time more sophisticated. A current-generation site not only has an overall theme for the site, but the organization of the information and the navigational scheme is based on a metaphor of the theme, called a Web Metaphor. A web metaphor is a design element that helps the user to leverage their existing experience in the abstract environment of the web. In this study, an approach to a thinking process model for web design is proposed through the concept of metaphors, and a theoretical analysis based on creative thinking for solving design problems is studied. This study aims to provide a glimpse into the world of metaphors and how they can be used in user interface design, to analyze selected examples, and to introduce a model web design process. As the study of the essence of creative thinking in web design is ongoing, diverse possibilities for solving problems in web design can be found through the theoretical modeling of thinking in web design. This study will also explore strategic and effective web design by establishing a mental model for web designers based on the study of metaphors that will inspire the thinking of designers.
\end{abstract}

Keywords: metaphor, web design, creative thinking

\section{Introduction}

Graphic designers in the 21st century need creative thinking and information power. The adult generation of design that has depended on knowledge of design and its techniques for ways of living has passed into a new design world that requires integrated knowledge and creative thinking in various fields. Thus, metaphors' role and function, which have become a basis for such integrated knowledge and ideas, are very important in the graphic design field. In the following paragraphs, I will describe this study's objectives.

First, graphic designers have increasingly extended and stressed the role of metaphors as a means of transferring lots of meaning using a simple expression. Designers have usefully applied metaphors for creating visual languages. Various design fields have been using metaphors, including in product design, advertising business, logo-interface design, interaction, and so on.

Second, because metaphorical expressions can transfer various meanings, different from direct and text-based phrases, and because they represent efficiency in information transmission, designers and others have long used them as an important way to communicate. As designers' creative thinking finds relationships in processes and products based on existing facts and concepts, since creative thinking is an extension of metaphors, designers can achieve their thinking process through metonymic expressions. However, it is difficult to use such ideas practically because there is no model for a systematic thinking process. That is, metaphors are still not sufficient for their usability and applicability.

Kay Youn, Assistant Professor, Seton Hill University. 
Designers can achieve the development of such a thinking process through expressing new visual languages, which leads to creating new meanings in various design fields, including cultures and environments.

As design delivers facts directly but also include aesthetics, the designers need creative thinking to solve problems. Designers can establish their creative thinking in a process involving their intuitive and their sensory thinking, in which the designers use metaphors to create their designs. Therefore, designers will be conducting studies on this issue. They find it very important to investigate the nature of design by studying metaphors to implement design as a creative means for improving the quality of design.

Therefore, in this study, I propose an approach to a designers' thinking process through the concept of metaphors and their theoretical analysis based on the creative thinking to solve design problems. As this thinking process's range is very large, we show the metaphors' limits in web design. The metaphor is an important aspect of research in the design field and we will study it through various cases. In addition, we have also conducted studies on the metaphor in web-based systems as a concept of interfaces that are a core in the digital age in aesthetic and digital engineering fields. In particular, the metaphor has a close relationship to web usability. Our intent in this study is to present possibilities in abundant expressions and analyses instead of designers' and users' primary thinking based on their intuitive and analogical inference in metaphors. In addition, we will explore strategic and effective web design by establishing a mental model for web designers based on web designers' metaphor studies in such a way that encourages designers' thinking.

\section{Correlation Between Web and Metaphor}

\section{Metaphors in Web Designs}

One can achieve to understand some phenomena or new subjects by using a different idea through the inference of a metaphor, that is, an intimation of what users watch and understand. For instance, Graphical User Interface (GUIs) used in digital contents are the products of metaphors ${ }^{1}$. Regarding the files in GUI, users naturally store these files to folders, in a similar approach to that of practical life, in a computer. For another example, in the desktop designs in Macintosh, Windows, and Linux operating systems, the word "desktop" itself is a metaphor, as people arrange their work tools and documents on their physical desks.

As mentioned above, computer desktops are an integration of various metaphors. In general, to use metaphors in GUI is to transfer the concept and characteristics of a computer system to users through language systems that enable intuitive thinking. Thus, the representative metaphors in web designs are presented as menus and icons for easy use. Therefore, designers can use metaphors as a way to visually structure users' emotions and consciousness (Lakoff \& Johnson, 1980). In particular, as the human cognitive system is based on a conceptual metaphor, designers desire to position metaphors in their web designs at the center (Eklund, 2006). Thus, web-based metaphors find a similarity between two different concepts and work to change a concept to a different one, one that has already been largely distributed into daily life and thinking activities. Here, the appropriateness between these two concepts depends on the concepts' similarity, and on how well web designers understand the interfaces based on users' empirical and practical knowledge. It is the same idea as the definition of web designers' creative thinking, who create new facts and concepts by finding a relationship between web design processes and products that are designed by the existing facts and concepts. Web design is essentially an extended concept of metaphors in which web designers' thinking process is

1 The first computer to popularize the desktop metaphor over the earlier command line interface was the Apple Macintosh in 1984. 
established as a metaphorical expression that can be recreated as the out-put, or the interfaces. The sensory and symbolic languages generated in web-design thinking can be considered as a metaphor language. Thus, the metaphor plays an essential role in solving problems that occur in web planning and interface design. In addition, metaphors are the proper visual language for web design thinking and play an important role in stimulating the creativeness in design through maximizing transfers of meaning to users and inspiring curiosity.

\section{Characteristics of Metaphor}

Metaphors are part of the creative process for producing new concepts in cultures. The expression "love is tears" has been socially adopted as well as has been the understanding of love being a sweet and pleasant thing. A mouse in a computer system is the same idea. In daily life, what people think and what they do are presented metaphorically through user interfaces in which the deletion of computer files is represented by the idea of putting trash into a trashcan.

Most things that people consider as actual reality are in fact composed of metaphors. Metaphors do not only represent a linguistic expression but also a more important relationship with non-linguistic regions. Thus, although the word metaphor originated from literature, designers can also use the concept for their visual thinking to include various meanings. Metaphors have been largely used in the fields of text, illustration, and layout, and users can also apply it as an element of identities. Here, the term "possibilities" indicates that metaphors are not a closed figure corresponding to a fixed meaning, but an open system that has room for the imagination to work. It is related to a concept of design that forms a meaning system for communicating, reproducing, experiencing, and searching social orders.

Metaphors are not limited to functioning as expressions, but they require social, political, and cultural thinking and activities. By enabling continuous thinking, metaphors play a role in providing richness to thinking. Metaphors for solving design issues can be expressed in all design processes and are able to simultaneously present designers' formative aesthetics. In addition, if designers use metaphors when applying their thinking systems, they can more easily perform their designs.

\section{Types of Metaphors}

We can describe metaphors in the aspects of structure, content, and design.

Structural metaphors. In the aspect of structure, metaphors have the form of "A is B" in which " $A$ " is the original concept and " $\mathrm{B}$ " is a medium or medium concept. The reason that we can accept this equality is due to the similarity in which "A"'s several meanings are related to those of "B". Here, we can use several metaphor expressions based on comparison, indication, allegory, contrast, parallel, and exaggeration.

Content metaphors. In the aspect of content, we can describe metaphors by investigating the degree of empirical recognition. This means that an image does not represent its meaning by itself but requires surroundings in which a metaphor takes its meaning through an interaction between the elements in these surroundings. Doing this provides an opportunity to open a new angle of vision. As a creative metaphor is presented by combining two very different concepts, which are never related to each other, the metaphor is unexpected.

Symbolic metaphors. Symbols and signs already have their own cultural backgrounds and are developed and changed continuously through metaphors. Signs are imperfect but successful in particular because their imperfect metaphors represent an understanding of their surroundings and interactions. 
In the case of signs, metaphors become a communication media and a visual language. As a visual language, metaphors involve visual transfer as an indispensable way to unify people with different points of view. The visual language can transfer information more effectively than any other media. Visual transfers are general international things. A person can recognize them without regard to any academic features and help in thinking because they are part of a static language through a sensitive activity of dynamic descriptions. Although such a visual language is less accurate than a linguistic communication, it can extend the width of thinking and the possibility in analyses (Kepes, 1995, p. 180).

Signs are classified into natural and formal signs. The "natural signs" are naturally formed by coupled relations in our experiences (for example, black clouds hint a sign of a typhoon or rain) and the "formal signs" represent an artificial structure for the purpose of pointing to something. Here, the latter is called a symbol. That is, it is an intentionally selected sign for transferring the meaning of a "symbol". Thus, the symbol is a sign, but all signs are not symbols according to this classification.

In general, signs are a medium of communication according to the objective actualization of images to other users. To usefully draw such visual elements in signs, designers need to process a story that others can understand and that arouses their interest in addition to the fact that designers need to create beautiful images, consistency in overall layouts, and connectivity in styles. The most excellent strategy for designers is to properly use a metaphor that has such applicability and functionality. Signs' meanings are not limited to the primary step but continue to the secondary step. However, there is a sign system in which the meaning is terminated in the primary step. Logical signs, like scientific abbreviations and sign systems used in mathematics and chemistry are determined as types of abbreviations and are terminated in the primary step.

However, these signs are not always terminated in their originally intended meaning. The number "7”, for example, some consider a lucky number, and a red traffic sign indicates a warning signal in other areas than traffic, such as in tobacco advertisements. Thus, the secondary, additional meaning, though initiated through the primary meaning, extends to the additional meaning as representing imaginary, expressive, and value-evaluated meaning dimensions. In such meanings, the same sign expression may transfer several meanings. The sign "blue jeans", for example, might mean just "blue jeans" as the primary meaning, but it has a few secondary, or additional meanings (making it a metaphor for these things), such as youthfulness, Western culture, freedom, exploitation, and so on.

We can classify metaphors into three different types: indexical, receptive, and symbolic metaphors, according to the classification by Charles Sanders Peirce's sign characteristics. We classify each metaphor according to a graphic expression method and motion-type based on its relationship with the subjects that will be reproduced in images.

Indexical metaphors. An indexical metaphor expresses the actual figure of the objects related to the subjects of pointing accidentally or existentially. Here, the metaphor is a part of the subjects to be pointed to, or it has a correlation to the subjects and cognitive things that the user needs to recognize. An expression that shows the entire shape of the Earth, or a part of it, in order to address the meaning of "globalism", is an example of an indexical metaphor.

Receptive metaphors. A receptive metaphor is an expression that represents a similarity using a metaphor, which has a certain similarity in the subjects of pointing, or a resemblance, like similar images. Here, things are similar but not perfectly the same. Thus, a metaphor that expresses the shape of the earth as an image for presenting "globalism" is a receptive metaphor. 
Iconic metaphors. An iconic metaphor has no relationship to the primary meaning subjects, thus they are different from indexical and receptive metaphors. They can be expressed using a specific engagement system. Using the word "world" to represent "globalism" is a type of iconic metaphor. However, these three metaphors are not strictly classified because these three concepts are not separate ideas but dependent concepts. Thus, their boundaries are varied according to different points of view.

Metaphors play the roles of a major device for transforming primary and planar meanings in signs to multi-dimensional meanings. Metaphors are an important means for designers to illustrate the characteristics, features, and concepts in design, and such a structure affects users' application capability and convenience in interfaces. Graphic designers can also present metaphors by their intuitive experiences and their inferences, based on experiences. They can also use metaphors to formalize their creative thinking and to solve design problems.

\section{Web Designers’ Creative Thinking Process}

Authors and Web designers perform their design work by configuring the design's goal. Here, they determine the design objectives and present the design's attributes as the final products. Designers create their designs in a prescriptive manner, using a solution-forced strategy (Lawson, 1980, p. 67). Therefore, a design concept is one of synthesis in a specialized precognition (Pugh, 1991, p. 68). Thus, design thinking is a mental capacity as a conceptual type for sophisticatedly solving design problems. As shown in Figure 1, creativity, which is a core element in design thinking for solving given problems, means a process that performs a new, valuable conception in various social fields. It can be expressed as creativity or expression ability.

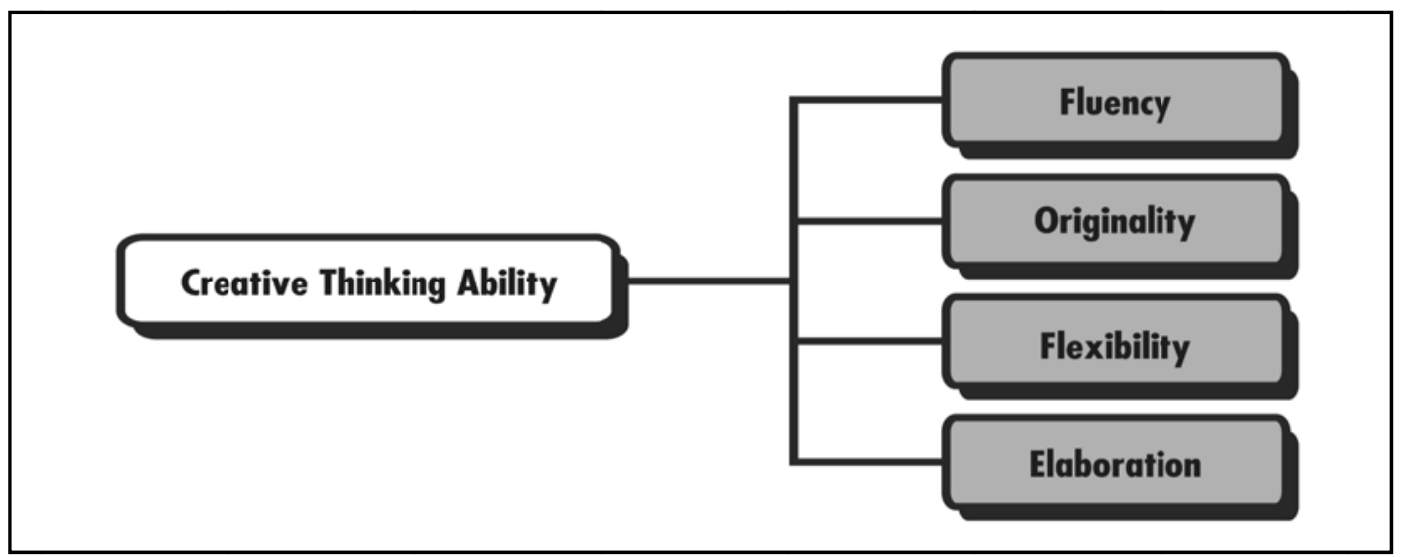

Figure 1. Model of creative thinking abilities.

The characteristic of creative thinking has a meaning of divergent thinking that draws as many answers as possible in the given condition, and differs from that of convergent thinking that draws a single answer in many given conditions. ${ }^{2}$ Also, as problems arise, creative designers create a new and meaningful conception.

\footnotetext{
2 J. P. Guilford (1897-1987) posited that a prime component of creativity is divergent thinking, the capacity to arrive at unique and original solutions and the tendency to consider problems in terms of multiple solutions rather than just one. Convergent thinking, which narrows all options to one solution, corresponds closely to the types of tasks usually called for in school and on standardized multiple-choice tests. In contrast, creativity tests designed to assess divergent thinking often ask how many different answers or solutions a person can think of to a specific question or problem. Some researchers have claimed that creative achievement actually involves both divergent and convergent thinking-divergent thinking to generate new ideas and convergent thinking to "reality test" them in order to determine if they will work.
} 
However, creative thinking can also be considered a complex type of reproducible thinking reminiscent of previous conceptions.

Web designers' creative thinking process begins when the designers understand there are issues in a web site planning that they must perform by analyzing directions, situations, and conditions for solving these issues. Also, they can implement benchmarking for solving problems by collecting and arranging information on other reference sites.

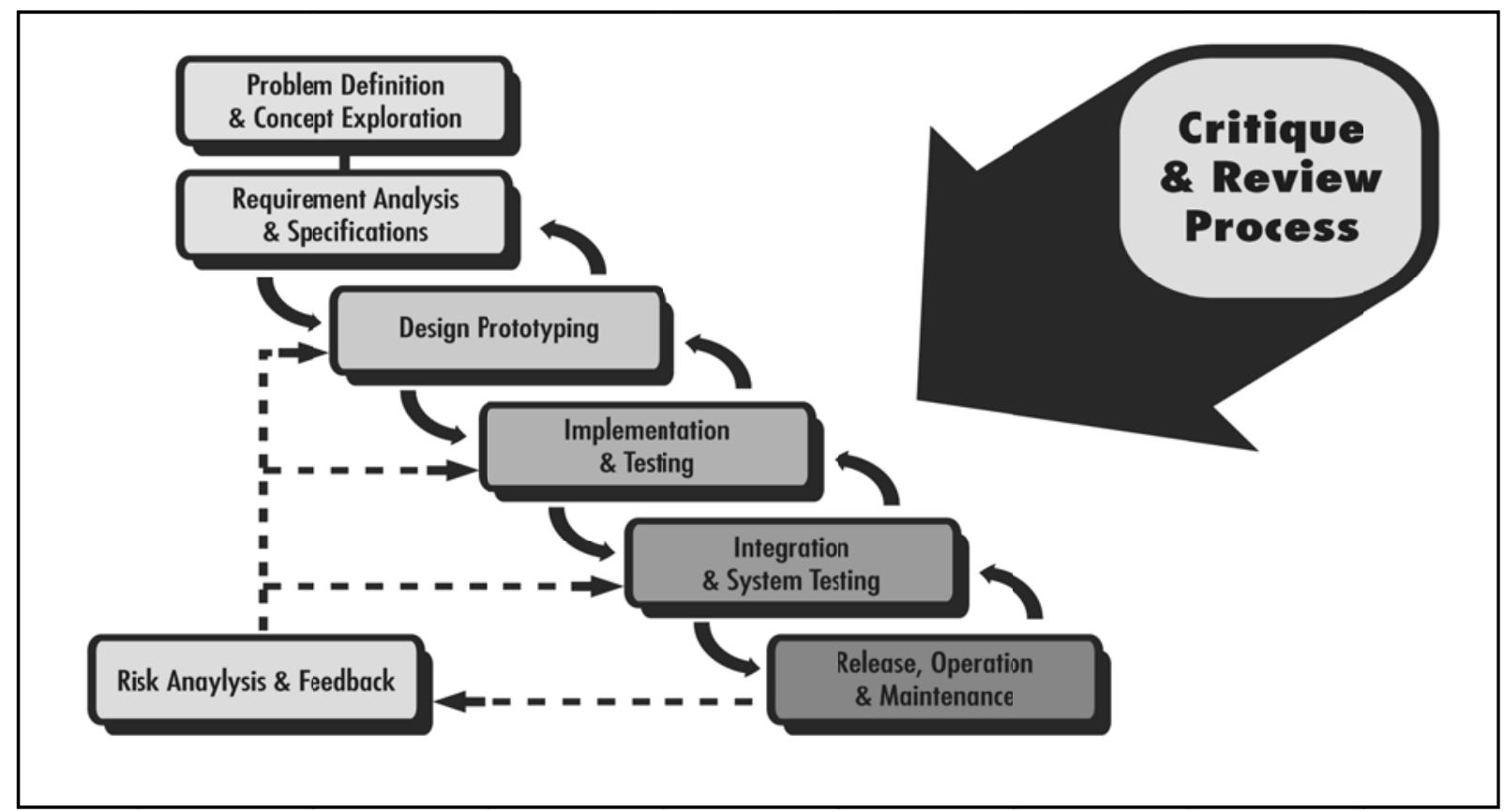

Figure 2. Conventional waterfall model of web process.

As shown in Figure 2, because the web design process may represent some problems in applying the conventional Waterfall Model, users must modify the model in order to obtain creative thinking capabilities. Thus, it is not easy to get a solution within a set timeframe, and the web designer must temporarily stop efforts to solve such problems when their thinking has been processed to a certain level or when they are implementing some activities that are not connected with the given problem. This is because they sometimes have unexpected inspirations or ideas after such a suspended period. However, they will need to apply some finishing touches and corrections to practically solve the idea's problems. Web designers' creative thinking process is summarized in the following four steps:

(1) A preparation period for recognizing problems and preparing methods;

(2) An incubation period for analyzing the problems' situations and conditions;

(3) A period for finding solutions;

(4) A period for implementing feedback and verifying the solutions.

Therefore, creativity in web design is a process that forms, transfers, and solves ideas or hypotheses through cognitive activities for presenting solutions in a web design process. Creative patterns are presented in creative activities in web designers.

The motivational force of web designers' creative thinking is basically initiated from a conception of ideas and an interest in projects, in addition to the concentration of continuous thinking. That is, web designers need 
an adventurous mind for overcoming some uncertainties on new sites, room for escaping and enjoying the conventional mold, and a confidence for developing their own idea without the distraction of other ideas, as well as an abundant imagination, reasonable association of ideas, flexibility in thinking, and close attention to details.

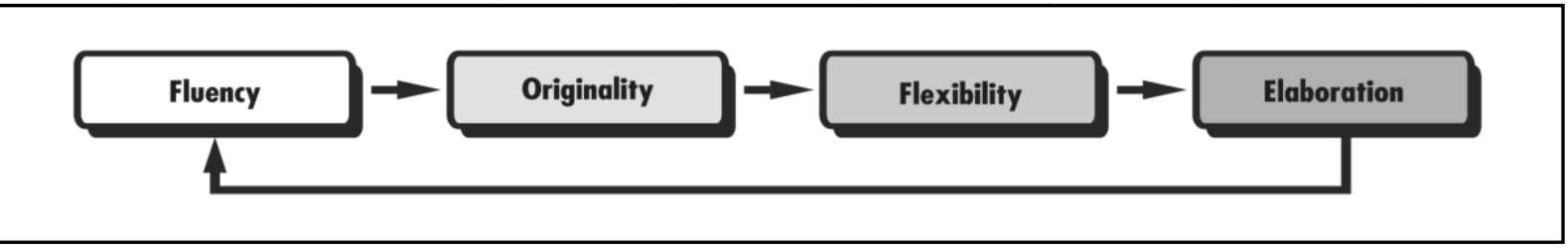

Figure 3. Web designers' creative thinking abilities.

One can measure web designers' creativity based on their fluency in language and expression, flexibility in considering new applications without constraining the conventional idea, originality, close attentions in planning, and redefinition. Web designers' creative thinking is eventually web-metaphor thinking.

Metaphor-based thinking becomes a basic tool for solving design problems and doing creative thinking. Visual thinking and visual cognition have a close relationship with each other. In particular, an idea sketch for presenting concepts and implementation is a typical case in graphic thinking.

Figure 3 shows that a solution to help the formation of a proper mental model of the web designer in web design is the use of visual and dynamic user-interface metaphors.

In particular, the metaphor in web design means an analogical inference model that minimizes the system that users will operate as well as the amount of specific learning they must undertake, through the designers' fast selecting a proper mental model according to how users will interface with the system. Here, the mental model is an intern expression for the conceptual understanding that users engaged in for some web sites.

\section{Cases Using Web Metaphors}

In web design, designers must undertake a reasonable thinking action prior to other things and they can arouse this thinking by a unique expression method. In this study, we will consider metaphor application cases that induce designers' thinking and users' activities in web design based on web sites and multimedia used in web sites.

\section{Case One}

Case one involves a genre of motion graphics that have been largely used in web sites and movies as a type of metaphor. This genre is a moving typography that Universal Film Company uses in the intros of its movies. In their movies, this typography represents an image of the coming Earth, and then the company title follows, in an effect that gradually illuminates the dark background (see Figure 4). The image of the earth coming out of darkness symbolizes coming out of ordeals and uncertainties into a bright world, thus representing the film company as a guide in the forthcoming story. It is a symbolic metaphor based on typical similarities and analogical inferences. 

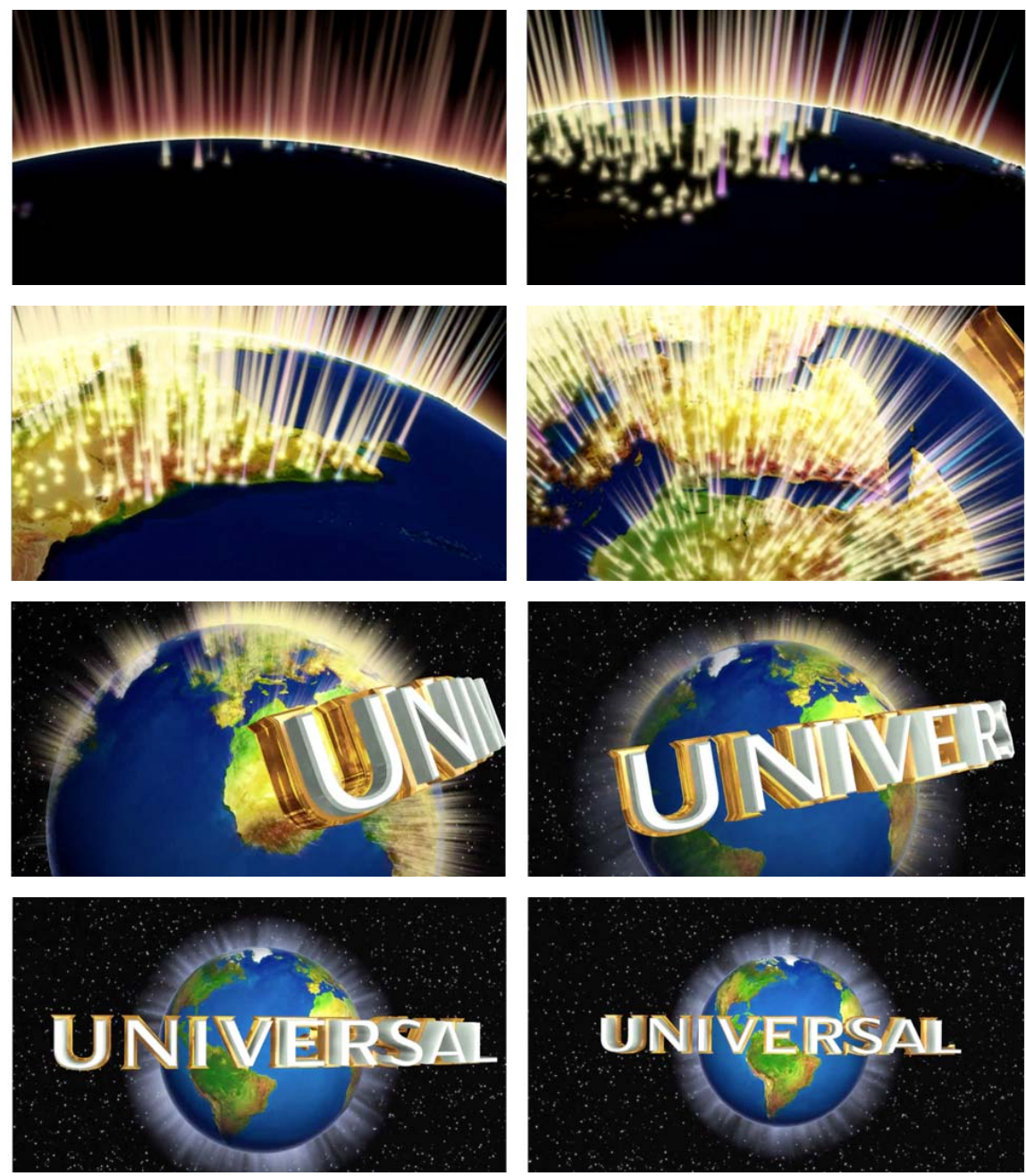

Figure 4. Universal Moving Typography: Intro Movie.

\section{Case Two}

Case two is a metaphor case using the image of "WEGA", a virtually-produced project. It shows dynamic screenplays and configurations in order to overcome the existing businesslike, stiff manners. In its introduction, different colors spread out in curved shapes from the center of the screen to the boundaries (see Figure 5). Then, the screen's center shows a small TV, and the rest of the TV gradually appears. In addition, a color board strip for controlling colors on a TV screen appears. After this color board disappears, a brilliant color flow comes out with the logos of WEGA and Sony one after the other. Sony and WEGA use metaphors with dynamic electric waves to symbolize familiar TV and other shapes to transfer their message.

\section{Case Three}

Case three involves the web site of an interactive company, Agencynet.com, which is located in Florida, USA (see Figure 6). The site shows an interface that configures environments and objects in a way as similar as possible to the real figures in order to improve the users' ability to see things intuitively. They use presentation metaphors for a daily-life environment that includes images of a large meeting room- "Clients", a small meeting room-“Capabilities”, a reception desk—“Contact Us”, a working room—“Portfolio”, a 
lounge_-“Philosophy”, and a recreation room-“Culture”. In particular, they have used a metaphor that mixes metaphor structures, representing objects and information spaces, and a structure metaphor to identify each information space. Also, the company has presented an inference-based metaphor that enables a design purpose and users to navigate this site intuitively, without any specific site-based learning. In addition, they have applied an esthetic metaphor for presenting visual appearances.

\section{Case Four}

In Case four, metaphor shows an interface used in iBooks apps, which is an Apple iPad application (see Figure 7). It uses both structure and presentation metaphors for presenting a bookstore in which the user can access the content of displayed books by mouse-clicking; the design is intuitive through a manipulation metaphor. That is, by using a metaphor that expresses detail and familiar concepts based on how users are likely to interact with information spaces, users can easily apply their predictions to the environment.
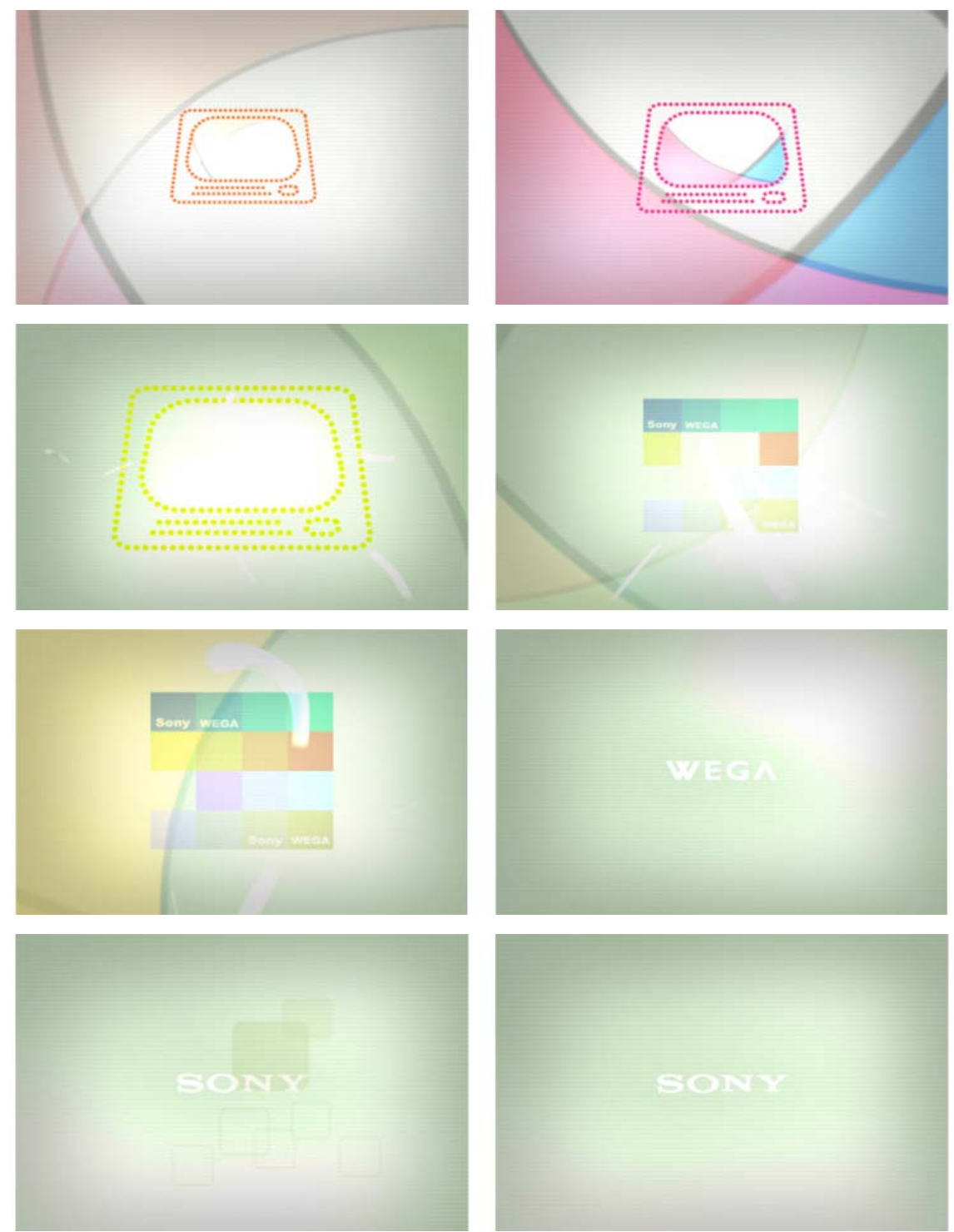

Figure 5. SONY/WEGA promotion movie introduction. 

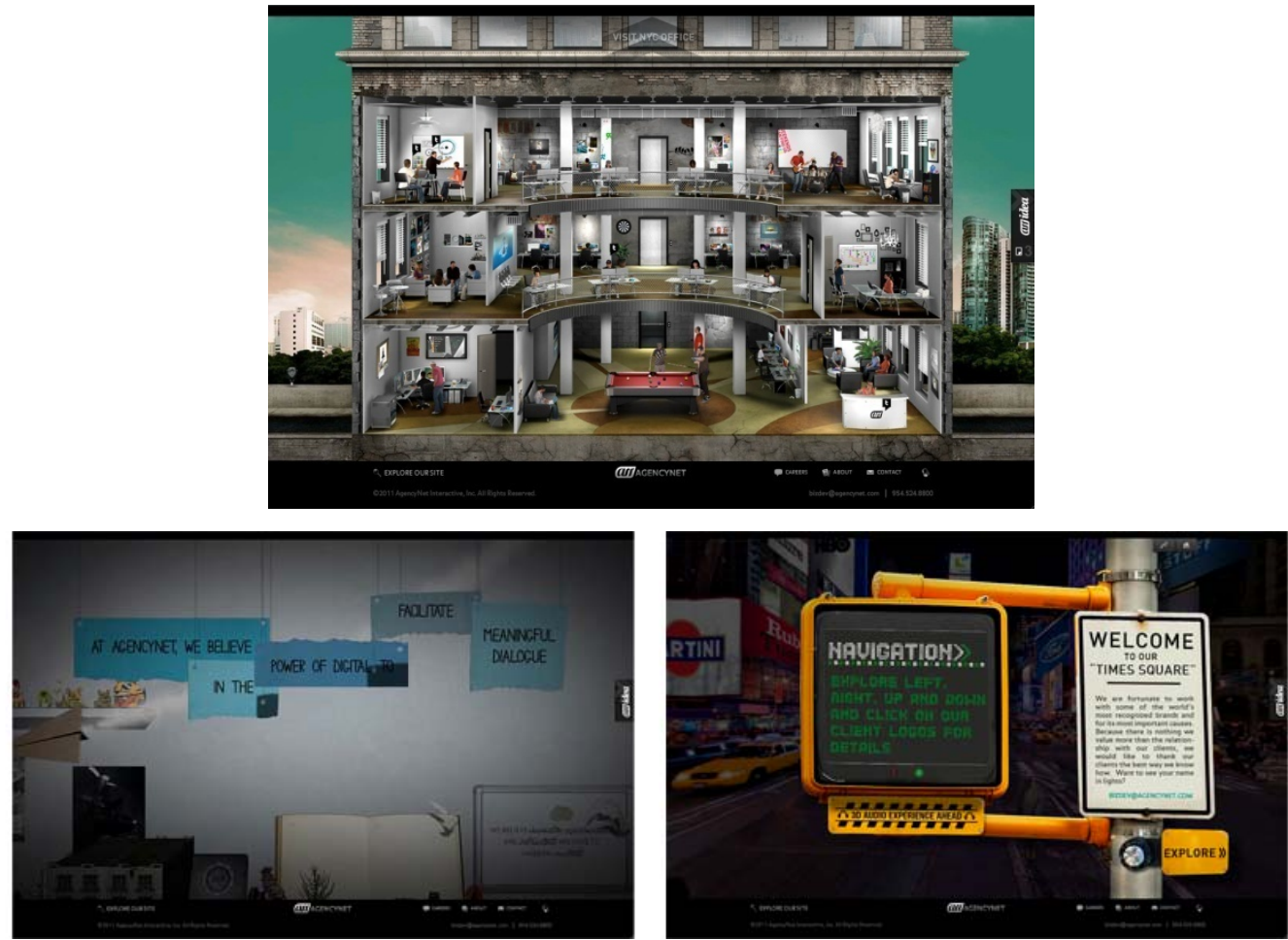

Figure 6. Feature of website. www.agencynet.com.

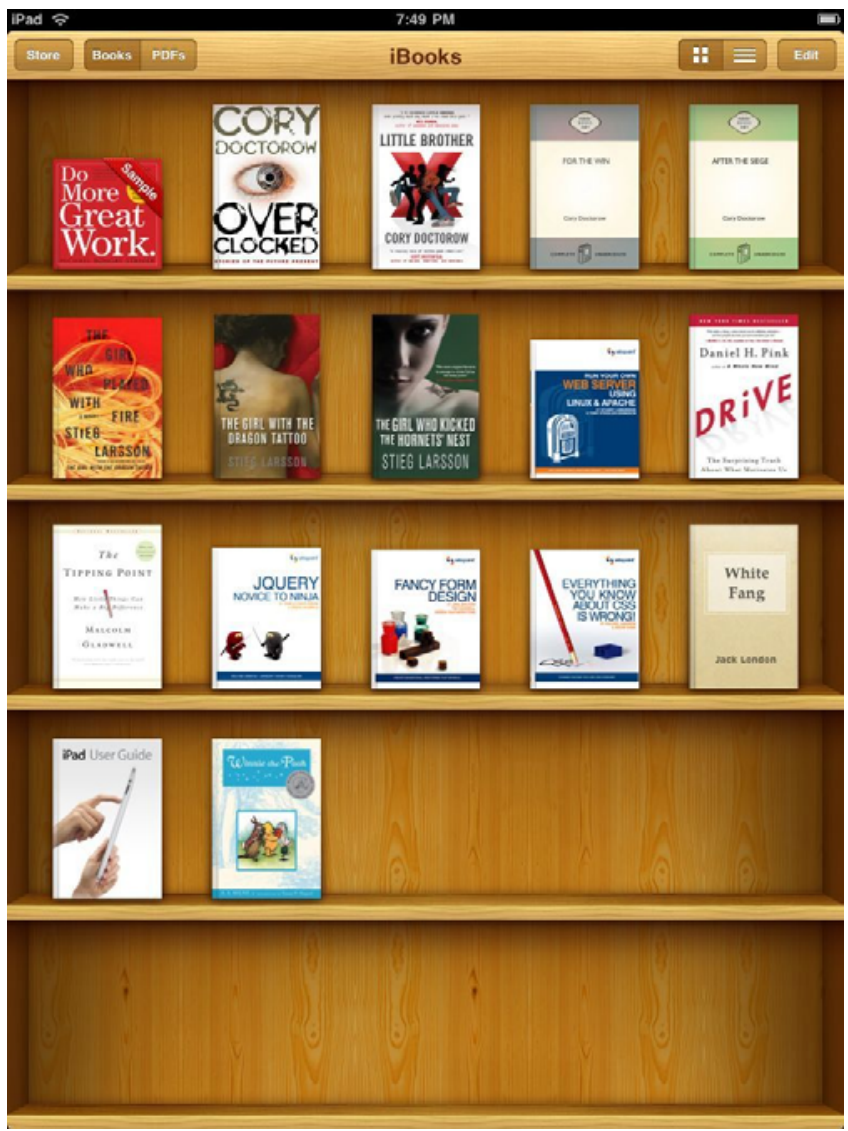

Figure 7. Feature of GUI.Apple iBook Application. 


\section{Case Five}

Case five represents a familiarity between users and subjects through a manipulation metaphor and encourages user-interaction as they must move a pen on the screen, followed by manipulating the mouse (see Figure 8). In this intuitive interface, we can illustrate a metaphor effect even though it uses metaphor expressions that affect users' applicability and the application convenience in interfaces.
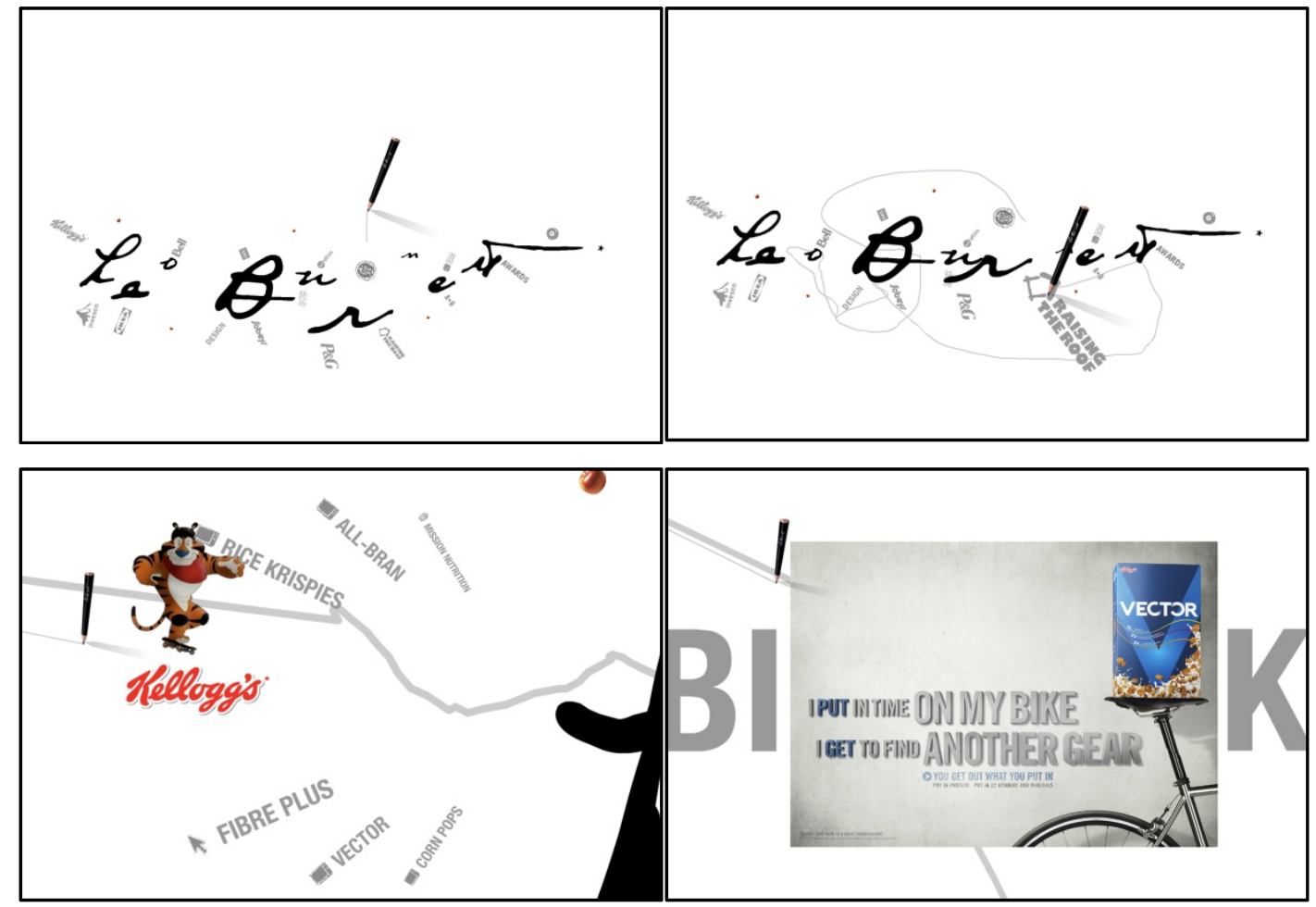

Figure 8. Feature of website. www.leoburnett.com.

Metaphors in web sites are GUIs, which can be defined as complex metaphors with graphic, terminology, touch, and hearing metaphors. Graphic metaphor means the overall image in web design, and Terminology metaphor represents letters and terms applied to menu bars and icons. Touch metaphor means a type of hardware metaphor such as a mouse, touch screen, text recognizer, and pens. And, hearing metaphor refers to non-oral sound interfaces such as rhythm, pitch, tone, sound frequency spectrum, and stress. That is, GUIs can be called a complex metaphor and a multimedia metaphor.

In the cases we have discussed in this study, we present an effect of web metaphors as the designers have used easy and intuitive interfaces, even though these metaphors use metonymic expressions. Also, an excessively sophisticated and complex interface system may present an opposite effect. These metaphors will play an important role in presenting the characteristics, features, and concepts of web design in which the structure of a metaphor significantly affects users' applicability and convenience of the interfaces' application. However, if web designers overstress the application of the metaphor based on their own intuitive thinking, they may create an obstacle in the web interfaces.

\section{Conclusion}

Designers require a capability that realizes what they think about various ideas effectively using visual or 
formative expressions. If they are capable of presenting things their users will recognize in the chosen imagines, their efforts in realizing these images will be an imaginative activity and will be the result of a complex and integrated mental creation process.

In this study, we attempted to outline an approach to web designers' creative thinking and the essence of design through four case studies of metaphors in web-based theoretical considerations and mental-thinking models. In addition, we analyzed the usefulness, applicability, and types of web metaphors.

These metaphors did not address their meanings with single images, but through the interaction between elements that compose an environment and that lead to good communication. Thus, metaphors have a close relation to the creative thinking in web designers and the designers can express their creative thinking through a web design process that includes their artistic sensitivity. We will extend metaphors' applicability and study it further through analyzing its complex relationship in various elements, such as sound, color, image, and so on, in addition to the visual elements in web design. In addition, if web designers' thinking system is improved using web metaphors, they can reduce the time and money they use in design tasks. It is necessary to follow this study with additional studies.

First, we will continue to conduct studies on the essence of design and creative design thinking.

Second, we will conduct studies on the establishment of theoretical models for design thinking and design processes.

Third, we will explore various possibilities for solving design problems through more systematic and stepwise studies based on these studies.

In addition, we have found it necessary to develop various design systems (DSs) that help designers' thinking and behavior processes and will develop a design expert system (DES) and a design strategic system (DSS).

\section{References}

Agencynet. (n.d.). Official company site. Retrieved June 12, 2011 from http://www.agencynet.com

Anderson, J. R. (1980). Cognitive psychology and its implications. San Francisco, CA: Freeman.

Duckner, E. (2002). Cross-cultural usability of the library metaphor. In Proceedings of JCDL, 223-230. Portland, OR: ACM Press.

Eklund, J. (2006). Cognitive models for structuring hypermedia and implications for learning from the world-wide web. Retrieved May 17 from http://ausweb.scu.edu.au/aw95/hypertext/eklund/

Familiant, E., \& Detweiler, M. (1994). Iconic reference: Evolving perspectives and an organizing framework. International Journal of Human-Machine Studies, 39(5), 705-728. London: Academic Press.

Gardiner, M. (1987). Applying cognitive psychology to user-interface design. Portsmouth: Wiley.

Gaver, W. (1989). The Sonic finder: An interface that uses auditory icons. Human-Computer Interaction, 4(1), 67-94.

Gaver, W. (1995). Oh what a tangled web we weave: Metaphor and mapping in graphical interfaces. CHI '95 Mosaic of Creativity (pp. 270-272). London: ACM Press.

Heckel, P. (1991). The elements of friendly software design. Alameda, CA: Sybex.

Kepes, G. (1995). Language of vision. New York: Dover Publication.

Lakoff, G., \& Johnson, M. (2003). Metaphors we live by (2nd ed.). Chicago: University Of Chicago Press.

Lawson, B. (1980). How designer think (4th ed.). Princeton: Architectural Press.

Leo Burnett. (n.d.). Personal promotion site. Retrieved June 20 from http://www.leoburnett.com

Luby, L. (1950). An introduction to logic. Philadelphia: Lippincott.

Maglio, P., \& Matlock, T. (1998). Metaphors we surf the web by introduction. Retrieved April 05 from http://www.ischool.utexas.edu/ i385e/readings/Magl

Marcus, A. (1998). Metaphor design in user interface. Journal of Computer Documentation, 22(2), 43-57. 
Marcus, A., \& West-Gould, E. (2000). Crosscurrents: Cultural dimensions and global web user interface design. ACM Interactions, 7(4), 32-46.

Pugh, S. (1991). Total design: Integrated methods for successful product engineering. London: Addison-Wesley Publication.

Shaikh, A. D., Chaparro, B. S., Nelson, W. T., \& Joshi, A. (2005). Metaphors and website design: A cross-cultural case study of the Tide,com stain detective. Usability News, $7(1)$. Retrieved July 03 from http://www.surl.org/usabilitynews/71/pdf/Usability\%20News\%2071\%20-\%20Shaikh.pdf

Smyth, M., Anderson, B., Knott, R., \& Altry, J. L. (1995). Reflections on the design of interface metaphors. Human Computer Interaction: Interact '95. Oxford: Alden Press. 